\title{
Implementación del Decreto Supremo 004-2013-PCM sobre la modernización de la gestión pública aplicada en la Municipalidad provincial de Huancayo
}

Implementation of the Supreme Decree 004-2013-PCM on the modernization of the public management applied in Huancayo provincial Municipality

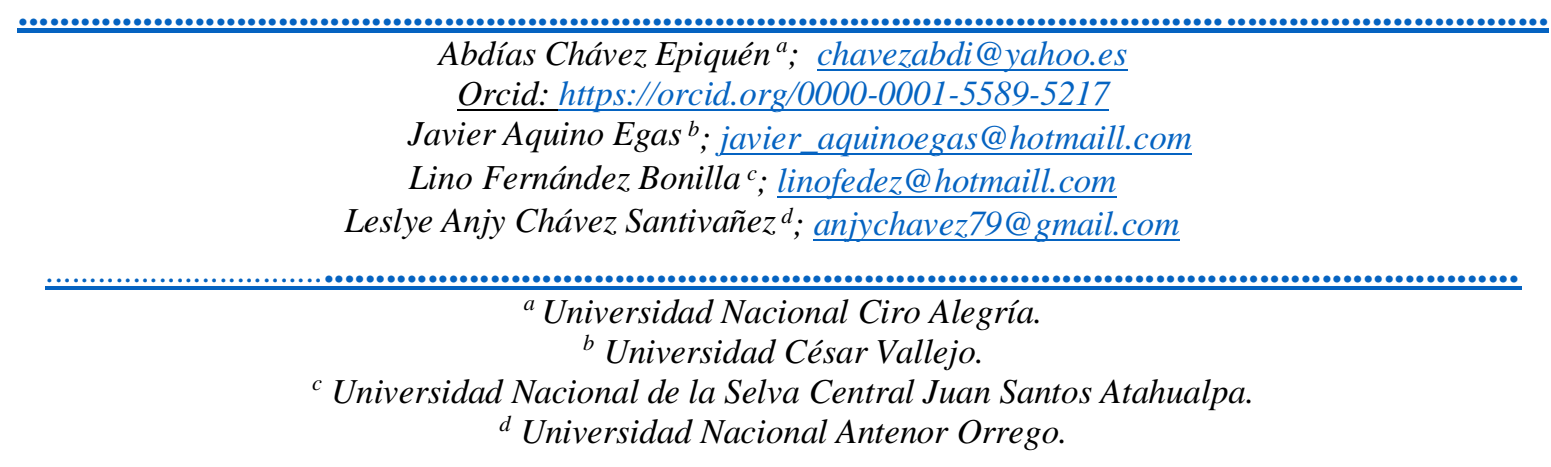

Recibido Julio/23/2020 • Aceptado: Agosto/02/2020 • Publicado: Septiembre/30/2020

\section{RESUMEN}

El objetivo con el que se presenta el trabajo de investigación es el de describir la aplicación de la Implementación del Decreto Supremo 004-2013-PCM sobre la modernización de la gestión pública aplicada en la Municipalidad provincial de Huancayo 2016, para conocer las características y niveles de la implementación de la Modernización de la Gestión Pública en la Municipalidad provincial de Huancayo 2016. La investigación, por su naturaleza fue de tipo básico y como métodos se utilizó el método científico, con un diseño descriptivo simple, para la muestra se tomó a los 72 trabajadores de la Municipalidad provincial de Huancayo. Se utilizó la técnica de la encuesta y el instrumento fueron los cuestionarios tipo escala Likert, sobre la variable Modernización de la Gestión Pública. Finalmente se concluyó, que la implementación y aplicación de la modernización de la gestión pública en la Municipalidad provincial de Huancayo en el periodo 2016 se encuentra en un nivel de inicio, desde la perspectiva de los servidores públicos; el 59,72\% perciben que la modernización de la gestión pública se encuentraen en un nivel de inicio en su aplicación; el 25.00 \% perciben que está en proceso; el $8.33 \%$ perciben que se encuentra en aplicación y el $6.94 \%$ manifestó que no se aplica esta norma en la institución pública.

Palabras claves: Modernización de la gestión pública, gobierno abierto, electrónico, interinstitucional.

a . Abdías Chávez - Epiquén; ${ }^{\mathrm{b} J a v i e r ~ A q u i n o ~-~ E g a s ; ~}{ }^{\mathrm{c}}$ Lino Fernández - Bonilla y d Leslye Anjy Chávez - Santivañez Articulo Protegido por Licencia Creative Commons: BY-NC-ND / Protected by Creative Commons: BY-NC-ND.

Sendas es una revista de acceso abierto / Sendas is an Open Access Journal. 


\section{ABSTRACT}

The aim with the one that appears the work of investigation was of describing the application of the Implementation of the Supreme Decree 004-2013-PCM on the modernization of the public management applied in Huancayo's provincial Municipality 2016, to know the characteristics and levels of the implementation of the Modernization of the Public Management in Huancayo's provincial Municipality 2016. The investigation, for his nature it was of basic type and as methods the scientific method was in use, besides the quantitative method, with a descriptive simple design, for the sample one took all 72 workers of Huancayo's provincial Municipality. There was in use the technology of the survey and the instrument they were the questionnaires type climbs Likert, on the variable Modernization of the Public Management. Finally one concluded, that the implementation and application of the modernization of the public management in Huancayo's provincial Municipality in the period 2016 is in a level of beginning, from the perspective of the public servants; $59,77 \%$ perceives that the modernization of the public management encuentraen a level of beginning in his application; $25.00 \%$ perceives that it is in process; $8.33 \%$ perceives that he is in application and $6.94 \%$ it demonstrated that this norm is not applied in the public institution.

Key words: Modernización of public administration, open, electronic, inter-government

\section{$\underline{\text { Introducción }}$}

La investigación tuvo la finalidad de describir la Implementación del Decreto Supremo 004-2013-PCM sobre la modernización de la gestión pública aplicada en la Municipalidad provincial de Huancayo; la variable fue estudiada siguiendo los procedimientos del método científico con el propósito de conocer su aplicación. El estudio permitió encontrar antecedentes de investigación acerca del tema; García (2007) publicó sobre, la nueva gestión pública: evolución y tendencias. Artículo publicado por la Secretaría General de Presupuestos y Gastos del Instituto de Estudios Fiscales de la Universidad de Salamanca. Entre las principales conclusiones se tiene que:

En las últimas décadas, las administraciones públicas de la mayoría de los países desarrollados se han visto inmersas en procesos de regeneración con el objetivo de conseguir una administración más eficiente y

a . Abdías Chávez - Epiquén; ${ }^{\mathrm{b}}$ Javier Aquino - Egas; ${ }^{\mathrm{c}}$ Lino Fernández - Bonilla y d Leslye Anjy Chávez - Santivañez Articulo Protegido por Licencia Creative Commons: BY-NC-ND / Protected by Creative Commons: BY-NC-ND. Sendas es una revista de acceso abierto / Sendas is an Open Access Journal. 
ágil que pueda adaptarse a las necesidades de los ciudadanos, sin que ello se traduzca en un incremento de la presión fiscal, sino que incluso se tienda a la contención o reducción del gasto público.

Las actuaciones desarrolladas son de diversa índole, pero básicamente se podrían distinguir dos grandes tipos, las que implican la apertura al mercado de ámbitos habitualmente sometidos a los procesos de gestión pública, y aquellas que suponen la adopción de las técnicas usadas por las empresas privadas en el ámbito público.

La utilización de técnicas privadas en el ámbito público ha supuesto el desarrollo de la dirección estratégica como técnica directiva y consecuentemente un importante auge de los sistemas de control interno de los entes públicos. Dentro de estos ha adquirido especial relevancia, la ampliación de los tradicionales procedimientos de verificación desarrollados en el ámbito de la información financiera hacia las auditorias operativas y el complemento de las tradicionales técnicas presupuestarias y contables con nuevas herramientas informativas de ayuda a la toma de decisiones como los indicadores de gestión.

Por otro lado Monteajeno, (2009) desarrolló la tesis, "modernización de la administración pública base del desarrollo empresarial en México". Se tuvieron las siguientes conclusiones: El principal problema del país no es la centralización, si no la corrupción; se requiere urgente desarrollar una cultura sistematizada constitucional de conciencia política nacional. El acto de corrupción ha corroído todo el sector empresarial, los modelos económicos y la sociedad. Los efectos alcanzan a la inversión, la empresa, el empleo, la producción, la confianza y lo sustancial las potencialidades de crecimiento.

Los resultados que se esperaban no han sido logrados debido a la paralización y ejecución de programas de modernización; escasa formación de profesionales y también por la improvisación de las acciones de gobierno, no rendición de cuentas e impunidad a los funcionarios responsables de corrupción.

Castillo, (2014), sustento la tesis, análisis del diseño de la política de calidad del aire de la municipalidad metropolitana de Lima. Tesis para optar el Grado de Magister en Ciencia Política y Gobierno, su análisis dio como conclusión; la Política Metropolitana del Ambiente vigente, aprobada por Ordenanza $\mathrm{N}^{\circ} 1628$ y la Política Ambiental Metropolitana aprobada por Ordenanza $\mathrm{N}^{\circ} 1424$ derogada,

a . Abdías Chávez - Epiquén; b Javier Aquino - Egas; ${ }^{\mathrm{c}}$ Lino Fernández - Bonilla y d Leslye Anjy Chávez - Santivañez Articulo Protegido por Licencia Creative Commons: BY-NC-ND / Protected by Creative Commons: BY-NC-ND. Sendas es una revista de acceso abierto / Sendas is an Open Access Journal. 
promueven el uso alternativo de transporte distinto al motorizado, caso del transporte peatonal y de bicicletas. Se considera que las alternativas planteadas en esta normativa no satisfacen la resolución del problema ya que las distancias a recorrer son muy amplias. Aun así, se toman en cuenta estas medias para la modernización del parque automotor, disminución de la contaminación del aire y menos uso de combustible.

Asimismo, Madrid (2011) investigó, "estudio de buenas prácticas en los compradores del sector público"; trabajo de tesis para el grado de magister sustentada en la Pontificia Universidad Católica del Perú. Cuyas conclusiones fueron; las mejoras en cuanto a las adquisiciones públicas radican en aprovechar los procesos internos implícitos a partir de la interpretación explicitan de las normas de cumplimiento obligatorio.

Existirán mayores posibilidades de éxito en la mejora de la gestión de las compras públicas, en la medida que se aprovechen al máximo las oportunidades o espacios de gestión que toda institución pública posee para introducir un conjunto de herramientas, utilizadas en mayor medida en el sector privado, que permitan mayores niveles de eficiencia y eficacia, sin dejar de lado el seguimiento de disposiciones legales ya explicitas ya elaboradas por el OSCE (Organismo Supervisor de la Contrataciones del Estado) .

Gutiérrez (2016) sustentó, modernización de la gestión pública y la gobernabilidad en la municipalidad distrital de Daniel Hernández de Tayacaja. Trabajo presentado en la Universidad César Vallejo para obtener el grado de magister en Gestión Pública. Los resultados concluyeron que existe una relación directa débil entre la modernización de la gestión pública y la gobernabilidad en la percepción del personal administrativo del distrito de Daniel Hernández. En la parte descriptiva se encontró que la gobernabilidad es alta, pero que la implementación de la norma no ha sido hecho realidad, es decir está en una etapa de inicio.

\section{MODERNIZACIÓN DE LA GESTIÓN PÚBLICA}

Tiene como origen el D.S. 004-2013 PCM, dentro de la Ley No 27658 - Ley Marco de Modernización de la Gestión del Estado que queremos llegar a que todos los peruanos sepan que cosa se hace en su comunidad ya sea local, regional y por consiguiente nacional, desarrollado de manera coordinada entre el

\footnotetext{
a . Abdías Chávez - Epiquén; ${ }^{\mathrm{b} J a v i e r ~ A q u i n o ~-~ E g a s ; ~}{ }^{\mathrm{c}}$ Lino Fernández - Bonilla y d Leslye Anjy Chávez - Santivañez Articulo Protegido por Licencia Creative Commons: BY-NC-ND / Protected by Creative Commons: BY-NC-ND. Sendas es una revista de acceso abierto / Sendas is an Open Access Journal.
} 
Sendas. 1(3), Julio - Septiembre, 2020.

URL: https://www.revistas.infoc.edu.pe/index.php/sendas

Email: revistasendas@infoc.edu.pe

Poder Ejecutivo, a través de la Secretaría de Gestión Pública y define la Modernización de la gestión pública "como una política de Estado con alcance a las entidades públicas en general no implicando los niveles de autonomía conferida por Ley. La norma ejerce para todos los organismos autónomos, organismo de gobiernos descentralizados, sociedad civil e instituciones políticas en sus diferentes organizaciones" todo esto gracias al Art. 118 inciso 8 de la Constitución Política del Perú que ante la necesidad de hacer algo y modernizar el Estado se crearon leyes establecidas como clara intención de dinamizarlo.

Carles R. (2009) sostiene que:

\section{"Las Administraciones} públicas son organizaciones a las que corresponde, satisfacer los intereses generales de sus respectivas comunidades". En el tenor del autor, una administración pública es una organización, por lo consiguiente todas las organizaciones con vinculo público.

La administración pública ha ido evolucionando, por los 60 tuvo un avance cualitativamente interesante a partir de la teoría de la organización
Vol. 1, $\mathrm{N}^{\circ} 3$, pp. 1-12 Septiembre 2020 ISSN $2708-6380$ https://doi.org/10.47192/rcs.v1i3.40

y la Teoría de sistemas, pues se interpretó que las organizaciones se den estudiar de modo integral. Las dimensiones fundamentales que acoge la administración pública son la estructura, entorno, objetivos, recursos humanos, tecnológicos, financieros y procesos.

Esta investigación se inicia tomando como base teórica a Moore, (1995) quien sostiene que:

Las intervenciones públicas se desprenden de la atención a las demandas de los ciudadanos; es decir, atendiéndose a problemáticas de interés público de la ciudadanía, las cuales se expresan a través de los mecanismos de decisión social. Centrándonos en el proceso político electoral del Poder Ejecutivo como mecanismo de decisión social, observamos que los representantes políticos, a través de los procesos electorales, asumen el compromiso de producir un conjunto de cambios en la calidad de vida de los ciudadanos, el cual buscará cumplir en el transcurso de sus mandatos. Las intervenciones públicas contienen así una promesa

a . Abdías Chávez - Epiquén; ${ }^{\mathrm{b}}$ Javier Aquino - Egas; ${ }^{\mathrm{c}}$ Lino Fernández - Bonilla y d Leslye Anjy Chávez - Santivañez Articulo Protegido por Licencia Creative Commons: BY-NC-ND / Protected by Creative Commons: BY-NC-ND.

Sendas es una revista de acceso abierto / Sendas is an Open Access Journal. 
de creación de valor público, dado que su atención a determinados problemas públicos se desprende de los objetivos gubernamentales, vinculados al compromiso de los representantes políticos.

Corrientes tan importantes como la Nueva Gestión Pública, han sido acogidas por estados latinoamericanos para trabajar reformas de modernización del Estado. A pesar de la fortaleza de las políticas de descentralización y del empoderamiento de los gobiernos locales, aún no están generalizadas las políticas de mejora de los servicios al ciudadano. Los gobiernos locales deben enfocar las reformas hacia una atención de calidad y hacia la entrega de servicios que satisfagan las necesidades de los ciudadanos.

Siguiendo con la propuesta de Pratsi Catalá (1998: 305) quien menciona que "las políticas públicas ya no pueden regirse más por la concepción unilateral de los administradores, como generalmente ocurría en la gestión burocrática clásica, sino que deben guiarse cada vez más por la satisfacción de los ciudadanos", podemos decir que nos encontramos en una etapa postweberiana donde el ciudadano se ha convertido no sólo en el fin supremo del Estado, sino en un actor https://doi.org/10.47192/rcs.v1i3.40

estratégico para el fortalecimiento de la tan ansiada gobernabilidad.

De hecho, hoy se encuentran valores en el eje del discurso de la administración pública contemporánea, vinculados a la relación Estado - ciudadano en donde es fundamental tomar en cuenta "la consideración del usuario de los servicios públicos como cliente, a cuya satisfacción se orienta la producción y a quien se le reconoce el derecho a exigir (...)" (Carlos Losada 2008: 299).

\section{Ejes transversales de la política de modernización}

Gobierno abierto: Políticas de gestión pública de amplio acceso y acercamiento a los usuarios de condición observable cuantificable y medible utilizando escalas de actitud.

Gobierno electrónico: Existencia y uso de TICS en los procesos de gestión pública de carácter medible utilizando escalas de actitud y cuestionarios

\section{Gobierno} interinstitucional: Estructura orgánica de la gestión pública aplicada en sistemas de interrelación con otras instituciones del estado o privada que opera posible de evaluar utilizando escalas de actitud y cuestionarios.

a . Abdías Chávez - Epiquén; ${ }^{\mathrm{b} J a v i e r ~ A q u i n o ~-~ E g a s ; ~}{ }^{\mathrm{c}}$ Lino Fernández - Bonilla y d Leslye Anjy Chávez - Santivañez Articulo Protegido por Licencia Creative Commons: BY-NC-ND / Protected by Creative Commons: BY-NC-ND. Sendas es una revista de acceso abierto / Sendas is an Open Access Journal. 
Servicio civil meritocrático:

Información sobre sistema institucional de procesos de inducción de recursos humanos que consiste en el reclutamiento, desarrollo, motivación y retención de personal.

\section{$\underline{\text { Metodología }}$}

Método general: Se aplicó el método científico, debido a que se trabajó en la realidad objetiva $y$ existe en forma independiente del sujeto, siguiendo un conjunto de procesos de rigor. La investigación por su naturaleza es del tipo Básica: Según Sánchez y Reyes, (1998) en estos estudios se describen los hechos y fenómenos tal como son y luego se intenta crear nuevas teorías o modificar las ya existentes.

Diseño de investigación: El diseño es No experimental - Transversal, denominado también descriptivo simple. Según Sánchez, (1998, p.79), “Estos diseños se orientan a la determinación y caracterización de la variable que solo se limita a mostrar tal como es de los fenómenos o eventos observados".

La población está constituida por los usuarios de la municipalidad provincial de Huancayo en el 2016. La muestra está constituida por 72 usuarios de la municipalidad provincial de Huancayo; el mismo que ha sido determinada de forma criterial por accesibilidad del investigador. Para la recolección de datos se ha utilizado la técnica de la encuesta definida como la técnica de obtención de datos mediante la interrogación a sujetos que aportan información relativa al área de la realidad a estudiar.

El instrumento que se utilizó fue la encuesta adaptada a la escala de actitud del tipo Likert, consistente en una batería de ítems redactadas como afirmaciones. Los datos fueron analizados por aplicando la estadística descriptiva donde se organizó los datos en baremos por rangos de; implementado, en proceso, inicio. Para hallar el grado de confiabilidad se utilizó el Alfa de Cronbach

\section{$\underline{\text { Resultados }}$}

De los 72 usuarios de la Municipalidad provincial de Huancayo encuestados, se pudo apreciar que los niveles de la variable; encuestadas, el 59,72\% perciben que la modernización de la gestión pública se encuentra en un nivel de inicio en su aplicación; luego 18 usuarios que representan el 25,00 \% perciben que la modernización de la gestión pública en la

a . Abdías Chávez - Epiquén; ${ }^{\mathrm{b} J a v i e r}$ Aquino - Egas; ${ }^{\mathrm{c}}$ Lino Fernández - Bonilla y d Leslye Anjy Chávez - Santivañez Articulo Protegido por Licencia Creative Commons: BY-NC-ND / Protected by Creative Commons: BY-NC-ND. Sendas es una revista de acceso abierto / Sendas is an Open Access Journal. 
Municipalidad provincial de Huancayo está en proceso; luego solo 6 usuarios que es el 8,33\% perciben que se encuentra aplicación y 5 usuarios que representa el $6,94 \%$ manifestó que no se aplica esta norma en la institución pública.

En la dimensión gobierno abierto, el $44,44 \%$ percibe el proceso de implementación del D.SN ${ }^{\circ}$ 004-2013 de Modernización de la Gestión pública se encuentra en proceso de inicio en la Municipalidad provincial de Huancayo. Acerca de la dimensión gobierno electrónico, el $49 \%$ de los usuarios perciben que se encuentra en proceso. En la dimensión Gobierno interinstitucional, el resultado es que cerca del $59 \%$ percibe que se encuentra en inicio. Acerca del servicio civil meritocrático $39 \%$ considera que se encuentra en inicio.

\section{Discusión}

La investigación tuvo como objetivo: Describir los procesos de implementación y aplicación de la modernización de la gestión pública en la Municipalidad provincial de Huancayo en el periodo 2016. El resultado obtenido fue que el 59,72\% perciben que la modernización de la gestión pública se encuentra en un nivel de inicio en su aplicación; el 25,00 \% perciben que la modernización de la gestión pública está en proceso; el 8,33\% perciben que se encuentra en aplicación y el 6,94 \% manifestó que no se aplica esta norma en la institución pública.

Para este contexto es meritorio citar a Pratsi Catalá (1998: 305) quien considera que "los principios de políticas públicas no deben ser asumidas como una concepción de carácter administrativo, modelo burocrático clásico, pues necesita estar orientado a la satisfacción de los usuarios". En la propuesta Weberiana estamos en una etapa en la que el ciudadano es el fin supremo del estado y actor estratégico para la gobernabilidad.

Contrastando estos resultados, se puede corroborar con la Tesis de Pérez (2011) La administración pública en el estado moderno: Enfoques teóricos para el análisis de la administración pública. Sustentada en la Universidad Nacional de La Plata, Argentina. Se encontró que:

La administración pública, lejos de ser una organización simple, presenta, por lo tanto, una gran complejidad entendida en el marco de gobierno, influyendo y determinando específicos resultados públicos que están en gran medida acorde a su forma de

a . Abdías Chávez - Epiquén; ${ }^{\mathrm{b}}$ Javier Aquino - Egas; ${ }^{\mathrm{c}}$ Lino Fernández - Bonilla y d Leslye Anjy Chávez - Santivañez Articulo Protegido por Licencia Creative Commons: BY-NC-ND / Protected by Creative Commons: BY-NC-ND. Sendas es una revista de acceso abierto / Sendas is an Open Access Journal. 
Sendas. 1(3), Julio - Septiembre, 2020.

URL: https://www.revistas.infoc.edu.pe/index.php/sendas

Email: revistasendas@infoc.edu.pe

funcionamiento interno e interacción con el contexto social que la rodea.

En otro orden de cosas, pudo observarse que doctrinas que hoy en día están en el habla cotidiana de los funcionarios públicos, en cuanto a cómo hacer las cosas, a quién elegir y qué hacer, no son tan nuevas y revolucionarias como se cree. Cada una de ellas, en su estado más general e inclusive en sus diferentes variaciones, pueden ser rastreadas a lo largo de la historia en diferentes países, e inclusive postulada por diferentes pensadores de las más variadas corrientes.

Por último, es conveniente recalcar que el surgimiento de cada doctrina, postulada como máxima para el accionar de la Administración pública, se relaciona con un momento histórico específico, donde diferentes grupos de interés vieron conveniente adoptarlas para llevar a cabo políticas que respondieran a sus necesidades. Por lo tanto, pareciera ser indispensable analizar el contexto de emergencia de las mismas, para poder comprender en mayor profundidad el verdadero sentido de cada una de ellas.

\section{Conclusiones}

Vol. 1, N ${ }^{\circ}$ 3, pp. 1-12 Septiembre 2020 ISSN $2708-6380$ https://doi.org/10.47192/rcs.v1i3.40

1. La investigación determinó que la implementación y aplicación de la modernización de la gestión pública DS. $N^{\circ}$ 004-2013 en la Municipalidad provincial de Huancayo en el periodo 2015-2016 se encuentra en un nivel de inicio, desde la perspectiva de los servidores públicos; el 59,72\% perciben que la modernización de la gestión pública se encuentraen un nivel de inicio en su aplicación; el $25,00 \%$ perciben que está en proceso; el $8,33 \%$ perciben que se encuentra en aplicación y el 6,94\% manifestó que no se aplica esta norma en la institución pública.

2. La investigación determinó la implementación y aplicación de la política de un gobierno abierto en el marco de la modernización de la gestión pública en la Municipalidad provincial de Huancayo en el periodo 2016 se encuentra en un nivel de INICIO desde la percepción de los trabajadores; el $44.44 \%$ perciben que la dimensión Gobierno abierto de la Modernización de la gestión pública se encuentra en un en nivel de inicio; el $40,28 \%$ percibe que se encuentra en

a . Abdías Chávez - Epiquén; ${ }^{\mathrm{b} J a v i e r ~ A q u i n o ~-~ E g a s ; ~}{ }^{\mathrm{c}}$ Lino Fernández - Bonilla y d Leslye Anjy Chávez - Santivañez Articulo Protegido por Licencia Creative Commons: BY-NC-ND / Protected by Creative Commons: BY-NC-ND.

Sendas es una revista de acceso abierto / Sendas is an Open Access Journal. 
Sendas. 1(3), Julio - Septiembre, 2020.

URL: https://www.revistas.infoc.edu.pe/index.php/sendas

Email: revistasendas@infoc.edu.pe

un nivel de proceso; el $15,28 \%$

perciben en aplicación; luego ningun

trabajador manifestó que "no se

aplica".

3. La investigación determinó que la implementación y aplicación de la política de un gobierno electrónico en el marco de la modernización de la gestión pública en la Municipalidad provincial de Huancayo en el periodo 2016, se encuentra en un nivel de INICIO; desde la percepción de los trabajadores, el 26,39\% perciben que la dimensión gobierno electrónico se encuentra en un nivel de inicio en su implementación, el 48,61\% perciben que se encuentra en proceso; el 2,78\% perciben que se encuentra en aplicación y el 22,22\% manifiesta que "no se aplica".

4. La investigación determinó que la Implementación y Aplicación de la política de un gobierno interinstitucional en el marco de la modernización de la gestión pública en la Municipalidad provincial de Huancayo en el periodo 2016; el $56,94 \%$ perciben que la dimensión gobierno interinstitucional se
Vol. 1, N ${ }^{\circ}$ 3, pp. 1-12 Septiembre 2020

ISSN $2708-6380$ https://doi.org/10.47192/rcs.v1i3.40

encuentra en un nivel de inicio en su implementación, el $23.61 \%$ perciben que se encuentra en proceso; el $13,89 \%$ perciben que se encuentra en aplicación y el 5,56\% manifiesta que "no se aplica".

5. La investigación determinó que la implementación y aplicación del servicio civil meritocrático en el marco de la modernización de la gestión pública en la Municipalidad provincial de Huancayo en el periodo 2016 está en un nivel de INICIO; la percepción de los trabajadores, el $38,89 \%$ perciben el Servicio civil meritocrático se encuentra en nivel de INICIO; el 33,33\% perciben que está en proceso; el 19,44\% perciben que está en aplicación; y el 8,33\% perciben que "no se aplica".

\section{$\underline{\text { Referencias }}$}

Balestrini, M. (1997) Como se elabora el Proyecto de Investigación,

Editorial BL Consultores Asociados. Caracas

Carles R. (2009) Organización y administración Pública. Ministerio

a . Abdías Chávez - Epiquén; ${ }^{\mathrm{b}}$ Javier Aquino - Egas; ${ }^{\mathrm{c}}$ Lino Fernández - Bonilla y d Leslye Anjy Chávez - Santivañez Articulo Protegido por Licencia Creative Commons: BY-NC-ND / Protected by Creative Commons: BY-NC-ND.

Sendas es una revista de acceso abierto / Sendas is an Open Access Journal. 
2020.

URL: https://www.revistas.infoc.edu.pe/index.php/sendas

Email: revistasendas@infoc.edu.pe

para las Administraciones

Públicas. Madrid.

Castillo, (2014), sustento la tesis "Análisis del diseño de la política de calidad del aire de la municipalidad metropolitana de Lima. Tesis para optar el Grado de Magister en Ciencia Política.

Decreto Supremo $\mathrm{N}^{\circ}$ 004-2013-PCM (2013) Política Nacional de Modernización de la Gestión Pública. Norma legal diario Oficial el Peruano. Consultado el 09 de noviembre de 2016. www.elperuano.com.pe/.

García I. (2007) La nueva gestión pública: evolución y tendencias. Artículo publicado por la Secretaría General de Presupuestos y Gastos del Instituto de Estudios Fiscales de la Universidad de Salamanca. España.

Gutiérrez J. (2016) Modernización de la gestión pública y la gobernabilidad en la municipalidad distrital de Daniel Hernández de Tayacaja.

Hernández, y otros (2010) Metodología de la investigación. $5^{\circ}$ Edición.
Vol. 1, N ${ }^{\circ}$ 3, pp. 1-12 Septiembre 2020 ISSN $2708-6380$ https://doi.org/10.47192/rcs.v1i3.40

México. Editorial Mc Graw Hill. Interamericana. Editores S.A. de C.V.

Hernández, C. Fernández, C., Baptista, P. (2006). Metodología de la investigación. $4^{\circ}$ Edición. México: Mc Graw- Hill.

Kerlinger, F. (1998). Investigación del comportamiento. 2da. Edición. México: Mc Graw - Hill UNMSM. LEY N 27658 (2002) Ley Marco de Modernización de la Gestión del Estado. Norma legal diario Oficial el Peruano. Consultado el 09 de noviembre de 2016. www.elperuano.com.pe/.

Losada C (2008) Los escenarios de la Gestión Pública del siglo XXI. Escuela de Administración Pública de Cataluña.Barcelona.

Madrid J. (2011) investigó. "Estudio de buenas prácticas en los compradores del sector público" Tesis para obtener el grado de maestría en la Pontificia Universidad Católica del Perú.

Mejía E. (2013) La investigación científica en educación. $2^{\circ}$ Edición. Editorial de la

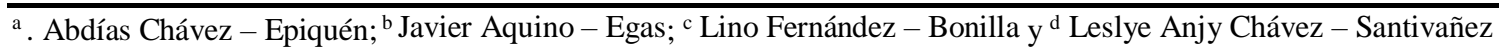
Articulo Protegido por Licencia Creative Commons: BY-NC-ND / Protected by Creative Commons: BY-NC-ND. Sendas es una revista de acceso abierto / Sendas is an Open Access Journal. 
Universidad Nacional Mayor de San Marcos. Lima Perú.

Monteajeno J. (2009) Modernización de la administración pública base del desarrollo empresarial en México; tesis desarrollada en el Instituto el Instituto Politécnico Nacional. Escuela superior de comercio y administración Unidad. Santo tomas México.

Moore, M. (1998) Gestión Estratégica y Creación de Valor Público en el Sector Público. Paidos, España.
Oseda, Chenet, Hurtado y Chávez (2014) Metodología de la Investigación. Editorial Soluciones Gráficas SAC. $5^{\circ}$ Edición. Huancayo Perú.

Pérez E. (2011) La administración pública en el estado moderno: Enfoques teóricos para el análisis de la administración pública.

Pratsi C. (1998) Administración pública y desarrollo en América Latina. Un enfoque Neoinstitucionalista. Revista CLAD Reforma y Democracia $\mathrm{N}^{\circ}$ Caracas.

\section{(c) 7 ()ㅜ}

Implementación del Decreto Supremo 004-2013-PCM sobre la modernización de la gestión pública aplicada en la Municipalidad provincial de Huancayo (Abdías Chávez - Epiquén) Por Revista Sendas se encuentra bajo una Licencia Creative Commons-No Comercial-Sin Derivadas 3.0 Uported.

a . Abdías Chávez - Epiquén; ${ }^{\mathrm{b} J a v i e r ~ A q u i n o ~-~ E g a s ; ~}{ }^{\mathrm{c}}$ Lino Fernández - Bonilla y d Leslye Anjy Chávez - Santivañez Articulo Protegido por Licencia Creative Commons: BY-NC-ND / Protected by Creative Commons: BY-NC-ND. Sendas es una revista de acceso abierto / Sendas is an Open Access Journal. 